\title{
A Phenomenological Study on Threshold Improvement via Spatial Coupling*
}

\author{
Keigo TAKEUCHI $^{\dagger \mathrm{a})}$, Toshiyuki TANAKA ${ }^{\dagger \dagger \mathrm{b})}$, and Tsutomu KAWABATA $^{\dagger \mathrm{c})}$, Members
}

\begin{abstract}
SUMMARY Kudekar et al. proved an interesting result in low-density parity-check (LDPC) convolutional codes: The belief-propagation (BP) threshold is boosted to the maximum-a-posteriori (MAP) threshold by spatial coupling. Furthermore, the authors showed that the BP threshold for code-division multiple-access (CDMA) systems is improved up to the optimal one via spatial coupling. In this letter, a phenomenological model for elucidating the essence of these phenomenon, called threshold improvement, is proposed. The main result implies that threshold improvement occurs for spatially-coupled general graphical models.

key words: spatial coupling, threshold saturation, belief propagation $(B P)$, dynamical systems.
\end{abstract}

\section{Introduction}

Low-density parity-check (LDPC) convolutional codes have been shown to outperform conventional LDPC block codes when iterative decoders based on belief propagation (BP) are used [1,2]. An LDPC convolutional code is constructed as a one-dimensionally coupled chain of LDPC block codes. As an explanation of this interesting result, it has been shown theoretically [3, 4] and numerically [5] that the BP threshold of an LDPC convolutional code is boosted to the maximum-a-posteriori (MAP) one of the corresponding LDPC block code. This phenomenon is called "threshold saturation" via spatial coupling [3,4].

Recently, we showed that the threshold saturation also occurs in spatially-coupled code-division multiple-access (CDMA) systems: The BP threshold for sparsely-spread CDMA systems is boosted to the optimal one via spatial coupling [6,7]. Since it is unclear whether the BP threshold for any graphical model can be improved to the optimal one, the term "threshold improvement" via spatial coupling is used in this letter, instead of threshold saturation. It is believed that threshold improvement is universal, i.e., the performance of the BP algorithm can be improved by coupling

Manuscript received January 1, 2011

Manuscript revised January 1, 2011.

${ }^{\dagger}$ K. Takeuchi and T. Kawabata are with the Department of Communication Engineering and Informatics, the University of Electro-Communications, Tokyo 182-8585, Japan.

${ }^{\dagger} \mathrm{T}$. Tanaka is with the Department of Systems Science, Graduate School of Informatics, Kyoto University, Kyoto 606-8501, Japan.

${ }^{*}$ The work of K. Takeuchi was in part supported by the Grantin-Aid for Young Scientists (B) (No. 23760329) from MEXT, Japan.

a)E-mail: ktakeuchi@uec.ac.jp

b)E-mail: tt@i.kyoto-u.ac.jp

c) E-mail: kawabata@ice.uec.ac.jp

DOI: 10.1587/transfun.E0.A.1 general graphical models spatially. This conjecture has been verified for several graphical models [8,9]. The purpose of this letter is to present a phenomenological study that supports the universality of threshold improvement.

Threshold improvement is a static property of spatiallycoupled graphical models, rather than dynamical properties. It is well-known that BP can calculate the MAP solution exactly if the factor graph defining the BP is a tree [10]. Furthermore, if the BP algorithm for a general factor graph converges, the BP fixed-points correspond to the stationary points of the so-called Bethe free energy for the factor graph [11], while the MAP solution corresponds to the global minimizer of the true free energy. Roughly speaking, the Bethe free energy is obtained by locally approximating the original factor graph by trees. These results allow us to characterize the static properties of the BP algorithm by stationary solutions to a dynamical system that has a potential energy function whose fixed-points coincide with those for the Bethe free energy, which is analogous to the density evolution (DE) equation for LDPC codes. In this letter, we restrict graphical models to such a class of graphical models that BP algorithms converge asymptotically.

As a phenomenological model for elucidating threshold improvement, we propose a spatially-coupled dynamical system with multiple stable solutions. This letter is organized as follows: In Section 2 the BP algorithm is characterized via a dynamical system with multiple stable solutions after presenting a motivating example for regular LDPC codes. In Section 3 a spatially-coupled dynamical system with multiple stable solutions is defined. Furthermore, an intuitive understanding of threshold improvement is presented. Section 4 presents the main result of this letter.

\section{Systems without Spatial Coupling}

\subsection{Density Evolution for regular LDPC Codes}

The explicit formula of the Bethe free energy for LDPC codes is unknown. We shall construct potential energy associated with the Bethe free energy from DE. Let us consider the DE equation for regular LDPC codes over binary erasure channel (BEC) with erasure probability $\epsilon[12$, Theorem 3.50]

$$
y_{t+1}-y_{t}=-\frac{d U}{d y}\left(y_{t} ; \epsilon\right), \quad y_{0}=1,
$$

where the potential energy associated with the Bethe free 
energy is given by

$$
U(y ; \epsilon)=\int_{0}^{y}\{z-\epsilon \lambda(c(z))\} d z
$$

with $c(y)=1-\rho(1-y)$. In these expressions, $y_{t}$ denotes the bit error rate (BER) for the BP decoder in iteration $t$. Furthermore, $\lambda(y)$ and $\rho(y)$ are given by $\lambda(y)=y^{d_{v}-1}$ and $\rho(y)=y^{d_{\mathrm{c}}-1}$, respectively, with $d_{\mathrm{v}}$ and $d_{\mathrm{c}}$ denoting the degrees of variable and check nodes. Note that the BP fixedpoint $y_{\infty}$ corresponds to a stationary point of the potential energy (2). When $\epsilon$ is smaller than the BP threshold $\epsilon_{\mathrm{BP}}$, the potential (2) has the unique stable solution $y_{1}=0$. This observation implies that the BER converges to zero in $t \rightarrow \infty$ for $\epsilon<\epsilon_{\mathrm{BP}}$. When $\epsilon>\epsilon_{\mathrm{BP}}$, on the other hand, the potential (2) has two stable solutions $y_{1}=0$ and $y_{\mathrm{r}}>0$, and one unstable solution $y_{\mathrm{u}}>0$, satisfying $y_{\mathrm{l}}<y_{\mathrm{u}}<y_{\mathrm{r}}$. The BER in this case converges to the strictly positive value $y_{\mathrm{r}}$ in $t \rightarrow \infty$. Threshold saturation [4] implies that $y_{t}$ can approach the smaller stable solution $y_{1}=0$ for $\epsilon \in\left[\epsilon_{\mathrm{BP}}, \epsilon_{\mathrm{MAP}}\right)$ by spatial coupling, with $\epsilon_{\text {MAP }}$ denoting the MAP threshold.

\subsection{Dynamical System with Multiple Stable Solutions}

In order to investigate the universality of threshold improvement, we consider a continuous-time dynamical system with a general potential energy function $U(y)$

$$
\frac{d y}{d t}=-\frac{d U}{d y}(y), \quad y(0)=y_{0} \in \mathbb{R} .
$$

As a simple example, let us consider a bistable potential energy function $U(y)$, shown in Fig. 11. The potential energy $U(y)$ has two stable solutions and one unstable solution $y_{\mathrm{u}}$. Let $y_{-}$and $y_{+}$denote the smaller and larger stable solutions, respectively, i.e., $y_{-}<y_{+}$. If the initial value $y_{0}$ is larger than the unstable solution $y_{\mathrm{u}}$, the state $y(t)$ converges to the larger stable solution $y_{+}$in $t \rightarrow \infty$. Otherwise, $y(t) \rightarrow y_{-}$. Without loss of generality, we assume that larger $y(t)$ implies better performance. The larger stable solution $y_{+}$corresponds to the optimal solution. Note that the term "optimal solution" is used to represent not the global stable solution but the largest stable solution in this letter. The typical BP solution corresponds to the smaller stable solution $y_{-}$, because the initial value for the BP algorithm is commonly smaller than the unstable solution $y_{\mathrm{u}}$, e.g., see [6,13] for CDMA systems. Thus, the inability of BP to get across the unstable solution makes the BP performance worse than the optimal performance when the potential energy $U(y)$ is not monostable.

In this letter, the potential energy $U(y)$ is assumed to have multiple stable solutions. We hereafter refer to the largest stable solution, denoted by $y_{+}$, and the other stable solution(s) as the optimal solution and the BP solution(s), respectively.

\section{Spatially-Coupled Dynamical System}

In order to obtain functionality of escaping from the BP solution(s), we consider a collection of identical systems coupled in a one-dimensional manner. Let $x \in\left(-x_{\max }, x_{\max }\right)$

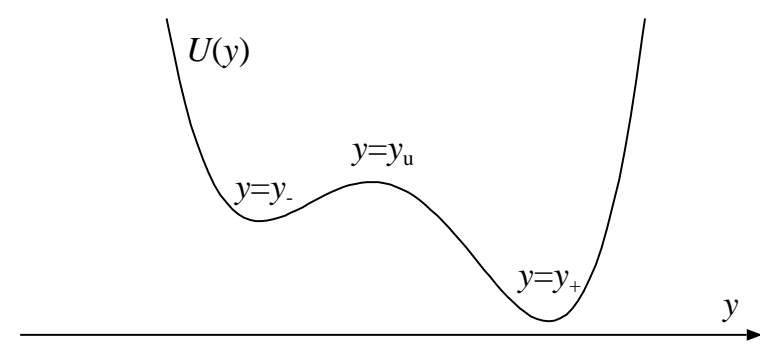

Fig. 1 Bistable potential energy $U(y)$.

denote the position of a system in the spatially coupling dimension, with $x_{\max }>0$ defining the size of the whole system, and let $y(x, t)$ be the state of the system at position $x$ and at time $t$. The spatially-coupled system we consider is governed by the equation

$$
\frac{\partial y}{\partial t}=-\frac{d U}{d y}(y)-\frac{1}{2} D^{\prime}(y)\left(\frac{\partial y}{\partial x}\right)^{2}+\frac{\partial}{\partial x}\left(D(y) \frac{\partial y}{\partial x}\right),
$$

where $D(y)>0$ is a positive coupling function. We study the system (4) with the initial and boundary conditions

$$
\begin{aligned}
& y(x, 0)=y_{0} \in \mathbb{R} \quad \text { for } x \in\left(-x_{\max }, x_{\max }\right), \\
& y\left( \pm x_{\max }, t\right)=y_{+} .
\end{aligned}
$$

Let $(2 N+1)$ denote the number of coupled systems. Threshold improvement occurs when the number of coupled systems, or equivalently $N$, tends to infinity and when the coupling strength $\|D(y)\|$ tends to zero [4,7]. The system (4) can be regarded as a space-continuum limit of $(2 N+1)$ space-discrete coupled systems in $N \rightarrow \infty$, or an approximation of finite differences in the coupled systems. These interpretations are justifiable by letting $\Delta=x_{\max } / N$ and considering $(2 N+1)$ copies of the original system at positions $x=i \Delta$ for $i=-N,-N+1, \ldots, N-1, N$. As $N$ gets large, the difference $y((i+1) \Delta, t)-y(i \Delta, t)$ is expected to be sufficiently small, so that one can approximate the difference by

$$
y((i+1) \Delta, t)-y(i \Delta, t) \approx \Delta \frac{\partial y}{\partial x}+\frac{\Delta^{2}}{2} \frac{\partial^{2} y}{\partial x^{2}} .
$$

The difference (7) is commonly included into a nonlinear function. Expanding the nonlinear function with respect to $\Delta$ yields a coupling function $D(y)$ depending on the state $y(x, t)$. See [7] for spatially-coupled CDMA systems. The last two terms on the right-hand side of (4) come from such approximations of space-discrete coupled systems.

Threshold improvement for spatially-coupled CDMA systems can be understood from (4) [7]. Unfortunately, the phenomenological model (4) does not include LDPC convolutional codes. This is because a higher-order approximation is needed near the boundaries for an ensemble of LDPC convolutional codes [4], while the DE equation can be approximated by (4) in the bulk region far from the boundaries.

The system (4) can be regarded as a dynamical system with the so-called Ginzburg-Landau free energy functional $H(y)[14]$ as its potential energy 


$$
\frac{\partial y}{\partial t}=-\frac{\delta H}{\delta y}(y)
$$

with

$$
H(y)=\int_{-x_{\max }}^{x_{\max }}\left[U(y(x, t))+\frac{D(y(x, t))}{2}\left(\frac{\partial y}{\partial x}\right)^{2}\right] d x .
$$

In (8),,$\delta / \delta y$ denotes the functional derivative with respect to $y$. The energy functional (9) implies that we impose spatial coupling that smooths the state $y(x, t)$ spatially. The point of spatial coupling is that the boundaries are fixed to the optimal solution $y_{+}$. A "stretched rubber rope," both ends of which are fixed to the optimal solution $y_{+}$, is utilized to "climb" the potential barriers between the BP solution(s) and the optimal solution. The tension of the rubber rope lifts the state $y(x, t)$ toward the optimal solution. In LDPC convolutional codes, such a boundary condition results from termination of convolutional codes [4].

\section{Main Result}

For simplicity, we hereafter assume that the coupling function does not depend on $y$, i.e., $D(y)=D>0$. We believe that the main result holds for state-dependent coupling functions. We focus on stationary solutions $y(x)$ to (4), satisfying

$$
0=-\frac{d U}{d y}(y)+D \frac{d^{2} y}{d x^{2}}, \quad y\left( \pm x_{\max }\right)=y_{+} .
$$

Figure 2 shows examples of the stationary solution $y(x)$ for the double-well potential $U(y)=y^{4} / 4-y^{2} / 2-h y$ with the parameter $h \in \mathbb{R}$. The double-well potential has a metastable solution $y_{-}<0$ (resp. $y_{+}>0$ ) and a stable solution $y_{+}>0$ (resp. $y_{-}<0$ ) for $h>0$ (resp. $h<0$ ). When $h=0.01$, the state approaches the uniform solution $y(x)=y_{+}$. When $h=-0.01$, on the other hand, the stationary solution $y(x)$ is a pot-shaped solution. Note that this solution is a natural solution for the case where the state $y(x, t)$ cannot climb potential barriers. Pot-shaped stationary solutions also appear in LDPC convolutional codes. See [4] for the details.

The main result of this letter is that there are no potshaped stationary solutions if the boundaries are fixed to the global stable solution.

Definition 1: A stationary solution $y(x)$ is called a potshaped solution if the following conditions are satisfied:

- $y(0)<y\left( \pm x_{\max }\right)$.

- $d y / d x \geq 0$ for $x>0$.

Note that any solution to (10) is an even function of $x$, because the differential equation (10) is invariant under the transformation $x^{\prime}=-x$. Thus, the second condition implies $d y / d x \leq 0$ for $x<0$.

Theorem 1: Suppose that the coupling function $D(y)>0$ does not depend on $y$. If the largest stable solution $y_{+}$of $U(y)$ is the unique global stable solution, then, there are no pot-shaped stationary solutions.

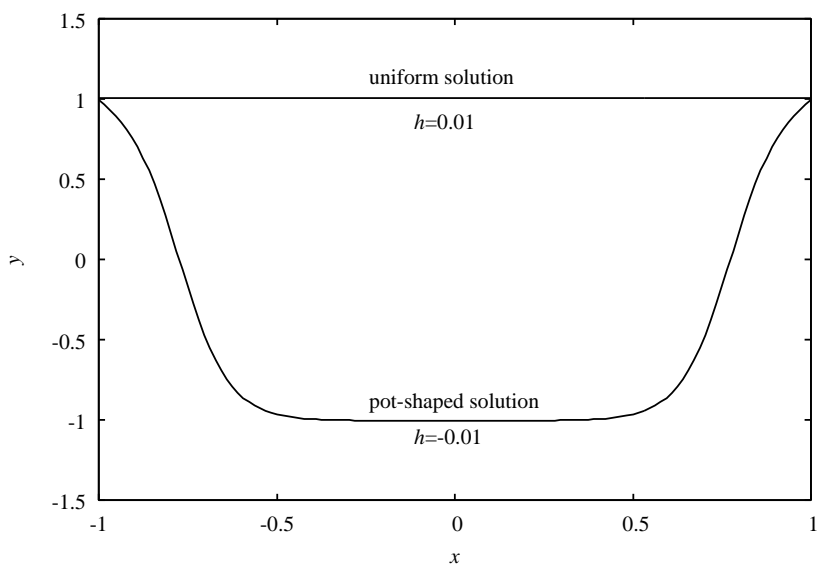

Fig. 2 Stationary solutions to (4) for $D=0.01$ and $x_{\max }=$ 1 . The initial value $y_{0}$ is equal to $y_{-}$.

Before proving Theorem 1 we shall present the significance of Theorem 11. Obviously, the uniform solution $y(x)=y_{+}$is a stationary solution to the spatially-coupled dynamical system (4). Intuitively, non-monotonic solutions on $x \in\left[0, x_{\max }\right]$ (or $x \in\left[-x_{\max }, 0\right]$ ) cannot become stationary solutions to (4) with the uniform initial condition $y(x, 0)=y_{0}$, because the state $y(x, t)$ should move closer to $y_{+}$as the position $x$ gets closer to the boundary. Thus, Theorem 1 implies that the state $y(x, t)$ converges to the uniform solution $y(x)=y_{+}$in $t \rightarrow \infty$ for any initial value, i.e., the state can approach the optimal solution for general potential energy $U(y)$ such that the largest stable solution $y_{+}$is the unique global stable solution.

Remark 1: Suppose that the continuum approximation of the DE equation(s) for a spatially-coupled graphical model is given by (4). We assume that the potential energy $U(y)$ is bistable and that it contains a parameter $\epsilon$, which corresponds to the erasure probability for LDPC codes over BEC or to the system load for CDMA systems. We rewrite the potential energy as $U(y ; \epsilon)$. The potential $U\left(y_{-} ; \epsilon\right)$ at the smaller stable solution $y_{-}$is assumed to be higher than that at the larger stable solution $y_{+}$for small $\epsilon$, as shown in Fig.11 As $\epsilon$ increases, the potential $U\left(y_{-} ; \epsilon\right)$ at the smaller stable solution is assumed to get lower, while the potential at the larger stable solution gets higher. Eventually, the potential energy $U(y ; \epsilon)$ may have the same height at the two stable solutions at $\epsilon=\epsilon_{\mathrm{BP}}^{(\mathrm{SC})}$. Theorem 1 allows us to define the $\mathrm{BP}$ threshold for the spatially-coupled graphical model as the point $\epsilon_{\mathrm{BP}}^{(\mathrm{SC})}$ at which $U\left(y_{-} ; \epsilon_{\mathrm{BP}}^{(\mathrm{SC})}\right)=U\left(y_{+} ; \epsilon_{\mathrm{BP}}^{(\mathrm{SC})}\right)$. The $\mathrm{BP}$ threshold $\epsilon_{\mathrm{BP}}^{(\mathrm{SC})}$ coincides with the optimal threshold for spatially-coupled CDMA systems [7]. It is unclear whether the BP threshold $\epsilon_{\mathrm{BP}}^{(\mathrm{SC})}$ coincides with the optimal one for any spatially-coupled graphical model.

Proof of Theorem $\square$, We shall prove Theorem 1 by contradiction. Suppose that there is a pot-shaped stationary solution $y(x)$. Integrating (10) after multiplying both sides by $d y / d x$, we obtain 


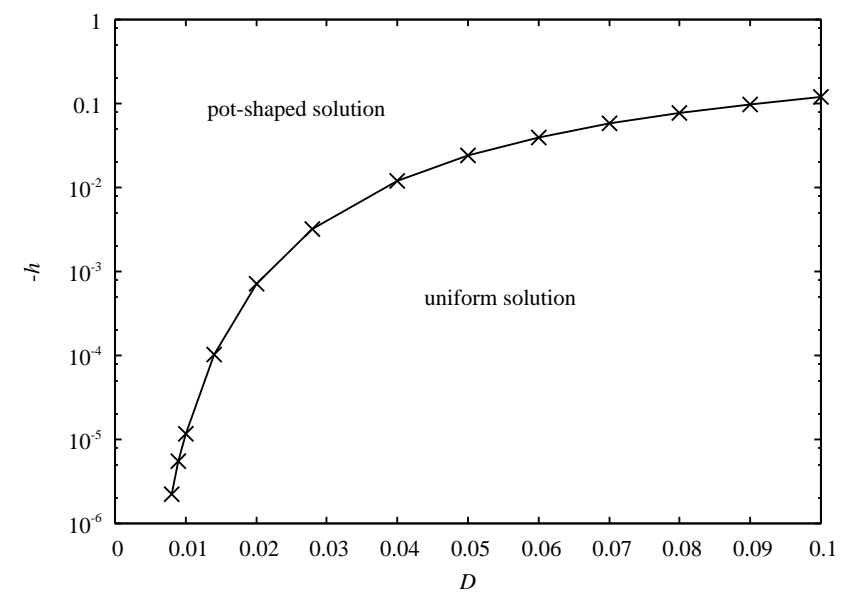

Fig. 3 Bifurcation diagram for $x_{\max }=1$, obtained by numerical simulations of (4). The initial value $y_{0}$ is equal to the smaller stable solution.

$$
\frac{D}{2}\left(\frac{d y}{d x}(x)\right)^{2}=U(y)+C
$$

with a constant $C$. We use the boundary condition $y\left( \pm x_{\max }\right)=y_{+}$and the positivity of the left-hand side of (11) to find $C \geq-U\left(y_{+}\right)$, where we have used the assumption that $y_{+}$is the unique minimizer of $U(y)$. If $y_{+}$was a local minimizer, the inequality would have to be replaced by $C \geq-U\left(y_{+}\right)+\Delta U$, with $\Delta U>0$ denoting the energy gap between $y_{+}$and the global minimizer.

Since we have assumed that there is a stationary solution satisfying $d y / d x \geq 0$ (resp. $d y / d x \leq 0$ ) for $x>0$ (resp. $x<0$ ), integrating (11) after taking the square root of both sides yields

$$
F(y)=x-\bar{x} \text { for } x>0,
$$

with

$$
F(y)=\sqrt{\frac{D}{2}} \int_{\bar{y}}^{y} \frac{d y^{\prime}}{\sqrt{U\left(y^{\prime}\right)+C}},
$$

where $\bar{y}$ denotes a value between $y(0)$ and $y_{+}$that does not minimize the potential energy, i.e., $U(\bar{y})>U\left(y_{+}\right)$. In (12), we have selected a constant of integration such that $y(\bar{x})=\bar{y}$. Repeating the same argument for $x<0$, we obtain

$$
y=F^{-1}(|x|-\bar{x}) \quad \text { for } x \in\left(-x_{\max }, x_{\max }\right),
$$

where $F^{-1}$ denotes the inverse function of (13).

Any stationary solution must be differentiable since it is a solution to the second-order differential equation (10). However, the solution (14) is not differentiable at the origin unless $d F^{-1} /\left.d y\right|_{y=y(0)}=0$ or $d F /\left.d y\right|_{y=y(0)}=\infty$, in which the value $y(0)$ at the origin is given by $y(0)=F^{-1}(-\bar{x})$. The uniqueness of the global stable solution implies that the integrand in (13) can diverge only at $y=y_{+}$. Thus, a necessary condition for $d F /\left.d y\right|_{y=y(0)}=\infty$ is $y(0)=y_{+}$, which contradicts $y(0)<y\left( \pm x_{\max }\right)$.
What occurs when the boundaries are fixed to a metastable solution? Figure 3 shows a bifurcation diagram of stationary solutions for the double-well potential $U(y)=y^{4} / 4-y^{2} / 2-h y$ with the parameter $h$. The larger stable solution $y_{+}$for $h<0$ corresponds to the metastable solution, i.e., the boundaries are fixed to the metastable solution. The state converges to a pot-shaped stationary solution in $t \rightarrow \infty$ when $(D,-h)$ is located above the curve shown in Fig. 3. Otherwise, the state converges to the uniform solution $y=y_{+}$. As long as $D$ is finite, the state may be conveyed to the uniform solution $y=y_{+}$for small $h$. However, such a solution seems to disappear in $D \rightarrow 0$, which corresponds to the limit in which threshold improvement occurs [4,7].

\section{References}

[1] A.J. Felström and K.S. Zigangirov, "Time-varying periodic convolutional codes with low-density parity-check matrix," IEEE Trans. Inf. Theory, vol.45, no.6, pp.2181-2191, Sep. 1999.

[2] A. Sridharan, M. Lentmaier, D.J. Costello, Jr., and K.S. Zigangirov, "Convergence analysis for a class of LDPC convolutional codes on the erasure channel," Proc. 42nd Allerton Conf. Commun., Control, \& Computing, Monticello, IL, USA, pp.953-962, Oct. 2004.

[3] S. Kudekar, T. Richardson, and R. Urbanke, "Threshold saturation via spatial coupling: Why convolutional LDPC ensembles perform so well over the BEC," Proc. 2010 IEEE Int. Symp. Inf. Theory, Austin, TX, USA, pp.684-688, Jun. 2010.

[4] S. Kudekar, T. Richardson, and R. Urbanke, "Threshold saturation via spatial coupling: Why convolutional LDPC ensembles perform so well over the BEC,' IEEE Trans. Inf. Theory, vol.57, no.2, pp.803-834, Feb. 2011

[5] M. Lentmaier and G.P. Fettweis, "On the thresholds of generalized LDPC convolutional codes based on protographs," Proc. 2010 IEEE Int. Symp. Inf. Theory, Austin, TX, USA, pp.709-713, Jun. 2010.

[6] K. Takeuchi, T. Tanaka, and T. Kawabata, "Improvement of BPbased CDMA multiuser detection by spatial coupling," Proc. 2011 IEEE Int. Symp. Inf. Theory, Saint Petersburg, Russia, pp.1629 1633, Aug. 2011.

[7] K. Takeuchi, T. Tanaka, and T. Kawabata, "Performance improvement of iterative multiuser detection for large sparsely-spread CDMA systems by spatial coupling," in preparation, 2011

[8] S.H. Hassani, N. Macris, and R. Urbanke, "Coupled graphical models and their thresholds," Proc. 2010 IEEE Inf. Theory Workshop, Dublin, Ireland, Aug.-Sep. 2010.

[9] K. Kasai and K. Sakaniwa, "Spatially-coupled MacKay-Neal codes and Hsu-Anastasopoulos codes," Proc. 2011 IEEE Int. Symp. Inf. Theory, Saint Petersburg, Russia, pp.752-756, Aug. 2011.

[10] J. Pearl, Probabilistic Reasoning in Intelligent Systems: Networks of Plausible Inference, Morgan Kaufmann, San Francisco, CA, 1988.

[11] J.S. Yedidia, W.T. Freeman, and Y. Weiss, "Constructing freeenergy approximations and generalized belief propagation algorithms," IEEE Trans. Inf. Theory, vol.51, no.7, pp.2282-2312, Jul. 2005.

[12] T. Richardson and R. Urbanke, Modern Coding Theory, Cambridge University Press, New York, 2008.

[13] Y. Kabashima, "A CDMA multiuser detection algorithm on the basis of belief propagation,” J. Phys. A: Math. Gen., vol.36, no.43, pp.11111-11121, Oct. 2003

[14] A. Schmid, "A time dependent Ginzburg-Landau equation and its application to the problem of resistivity in the mixed state," Phys. Kondens. Materie, vol.5, no.4, pp.302-317, Oct. 1966. 